\title{
Impact of smoking on tooth loss in adults
}

\author{
Abstracted from
}

Dietrich T, Walter C, Oluwagbemigun K, Bergmann M, Pischon T, Pischon N, Boeing H.

Smoking, Smoking Cessation, and Risk of Tooth Loss: The EPIC-Potsdam Study.

J Dent Res 2015; 94: 1369-1375. doi: 10.1177/0022034515598961.

Address for correspondence: T. Dietrich, Department of Oral Surgery, The School of Dentistry,

University of Birmingham, St. Chad's Queensway, B4 6NN, UK. E-mail: t.dietrich@bham.ac.uk

\section{Question: What is the association between smoking and tooth loss?}

\section{Design Cohort study}

Cohort selection Participants were recruited between 1994 and 1998 from the general population with the preferred ages of 35 to 65 years in women and 40 to 65 years in men.

Exposure measurement Smoking was assessed using a questionnaire from which pack years of smoking were calculated. Educational attainment, body mass index, hypertension, diabetes, alcohol consumption and vitamin or mineral supplements were assessed from measurements and questionnaires. Tooth loss was also assessed by questionnaire returned between 2004 and 2006. With the exception of the tooth loss data analysis was based on data collected at baseline. Data analysis The 24,373 participants who returned the tooth loss questionnaire were analysed. Two hundred and eighty-six (1.2\%) were excluded, as they did not respond to either of the tooth loss questions, and an additional 106 (0.4\%) were excluded because they gave inconsistent responses to the questions on tooth loss. Four hundred and thirteen (1.7\%) participants with missing data on cigarette smoking and $192(0.8 \%)$ participants with missing data in any of the covariates were also excluded. The association between smoking and number of teeth at baseline was assessed using negative binomial regression models to obtain relative risks and $95 \%$ confidence intervals (Cls).

Results The sample of 23,376 included 9,032 men and 14,344 women of which 4,394 (19\%) were current cigarette smokers, and 7,268 (31\%) were cigarette smokers. 1,566 (6.7\%) were edentulous at baseline. Compared with never smokers, current smokers were more likely to be male, less educated, more likely to be hypertensive, and less likely to take vitamins/mineral supplements, and they had higher alcohol consumption. Cigarette smoking was associated with higher prevalence of tooth loss at baseline as well as higher incidence of tooth loss during follow-up. The association between cigarette smoking and incident tooth loss during follow-up for the fully adjusted

\begin{tabular}{l|l|l} 
& $\begin{array}{l}\text { Male } \\
\text { Odds ratio }(\mathbf{9 5} \% \mathbf{C l})\end{array}$ & $\begin{array}{l}\text { Female } \\
\text { Odds ratio }(\mathbf{9 5} \% \mathbf{C l})\end{array}$ \\
\hline $\begin{array}{l}\text { Current smoker } \\
<15 \mathrm{cig} / \mathrm{d}\end{array}$ & $1.69(1.30$ to 2.21$)$ & $1.74(1.51$ to 2.01$)$ \\
\hline $\begin{array}{l}\text { Current smoker } \\
>15 \mathrm{cig} / \mathrm{d}\end{array}$ & $3.64(3.00$ to 4.42$)$ & $2.47(2.11$ to 2.89$)$ \\
\hline
\end{tabular}

model (adjusted for age, sex, education, diabetes, body mass index, waist-to-hip ratio, hormone replacement therapy, contraception, intake of vitamin and mineral supplements, physical activity, alcohol intake, hypertension, and cardiovascular disease) is shown in the table. Conclusions There is a strong dose-dependent association between cigarette smoking and the risk of tooth loss. The risk declines after cessation of cigarette smoking; however, the risk may remain elevated for up to 20 years compared with never smokers. Efforts to improve the oral health of the population should include the prevention of smoking as well the promotion of smoking cessation.

\section{Commentary}

Tooth loss is amongst the most prevalent oral conditions behind dental caries and periodontal disease. ${ }^{1}$ Recent estimates suggest that $2.3 \%$ of the global population are edentate, which equates to around 158 million people world-wide; this prevalence increases with age and peaks at approximately 60 years old. ${ }^{2}$ Tooth loss is the reflection of a lifetime of dental disease and treatment and is a complex outcome to measure. Therefore, it is important to understand the causes of, and possible ways to prevent, tooth loss in the adult population.

This observational study explores the association between cigarette smoking, smoking cessation and tooth loss within the adult population in Germany. The study has a cohort type design with longitudinal follow-up of adults recruited as part of a wider European Prospective Investigation into Cancer and Nutrition (EPIC). ${ }^{3}$ The main outcome measure, tooth loss, was assessed through baseline examination and self-reporting of teeth present in a follow-up questionnaire. Those who did not respond to dental questions, gave inconsistent responses to dental questions, had missing data on smoking or had any of the recorded EPIC covariates missing were excluded from the study.

The study is based on results for 23,376 adults, with a mean age of 50 years (ranging from 20 to 70 years), who were dentate and had complete data available for the measurements selected for analysis. This represented $95 \%$ of the original sampling frame and introduces a small but potential initial sampling bias. Those excluded due to incomplete data on the measurements selected could be representative of a group with specific characteristics from the population. Self-reported tooth loss has limitations as an outcome variable in that it is potentially prone to recall bias. It is easily over or underestimated and so could be considered a relatively unreliable measure. 
The cohort study design is used to test the hypothesis that the incidence and prevalence of tooth loss differs between adults who have never smoked, have given up smoking or who smoke. Multiple measured confounders are explicitly defined within the paper and accounted for within the analysis. These include age, sex, socioeconomic position as measured by participant education level and a variety of nutritional and medical markers collected as part of the wider EPIC study. The paper conforms to the STROBE guidance which provides recommendations on what should be included in the reporting of an observational study. ${ }^{4}$

A type of generalised linear regression called negative binomial regression is used to model the baseline association between smoking status and number of teeth present. The mean number of teeth present in the 'never', 'former' and 'current' smoking groups including \pm Standard Deviation were: $22.4 \pm 8.9,21.5 \pm 9.5$ and 20.5 \pm 9.9 respectively. Further full case analysis was carried out on these three categories for those participants who had at least one natural tooth remaining at baseline. Age and time between examination and the self-reported questionnaire were adjusted for in the basic model. Subsequent models then gradually adjusted for potential confounders. Finally, the association between smoking and tooth loss was considered for sex, and then for three categorical age groups. The results are reported as odds ratios which the authors advise to interpret as 'the relative risk of a tooth being lost as a function of smoking exposure, adjusted for covariates'.

This study ultimately reports that there was a consistent association between cigarette smoking and tooth loss which did not change by sex, age, education or other measured confounders.

The investigators have been successful in reducing, but not eliminating, confounding within this study. They address several limitations of the study within their discussion including the lack of information available on oral health and dental care. They also acknowledge that oral hygiene status and practices may differ between smokers and non-smokers.

Overall this study has provided some evidence of an association between smoking and eventual tooth loss. This is in addition to previous evidence linking smoking to periodontal disease. The primary clinical benefit which could be drawn from this study is the re-assertion of the importance of smoking cessation and the prevention of periodontal disease within the adult general population. In the United Kingdom, the prevalence of smoking is estimated to be $19 \%$ with higher rates in deprived populations. ${ }^{5,6}$ It is important that dental teams remain conscious of discussing tobacco use and its implications for both general and dental health with their patients.

Susan J Carson a and Jacqueline Burns ${ }^{b}$ ${ }^{a}$ Dental Health Services Research Unit, School of Dentistry, University of Dundee, Scotland ${ }^{b}$ Department of Public Health, NHS Fife, Scotland

1. Marcenes W, Kassebaum NJ, Bernabé E, et al. Global burden of oral conditions in 1990-2010: a systematic analysis. J Dent Res 2013; 92: 592-597.

2. Kassenbaum NJ, Bernabé E, Dahiya M, Bhandari B, Murray CJ, Marcenes W. Global Burden of Severe Tooth Loss: A Systematic Review and Meta-analysis. J Dent Res 2014; 93: 20S-28S.

3. Boeing H, Korfmann A, Bergmann MM. 1999. Recruitment procedures of EPICGermany. European Investigation into Cancer and Nutrition. Ann Nutr Metab 1999; 43: $205-215$.

4. Von Elm E, Altman DG, Egger M, Pocock SJ, Gøtzsche PC, Vandenbroucke JP; STROBE Initiative. The Strengthening the Reporting of Observational Studies in Epidemiology (STROBE) statement: guidelines for reporting observational studies. PLoS Med 2007; 4: e296.

5. Office for National Statistics. Statistics on Smoking England 2015. Health and Social Care Information Centre, $29^{\text {th }}$ May 2015. http://digital.nhs.uk/catalogue/PUB17526/ stat-smok-eng-2015-rep.pdf [Accessed 25 August 2016]

6. Action on Smoking and Health (ASH). ASH facts at a glance Smoking Statistics. June 2016. http://www.ash.org.uk/files/documents/ASH_93.pdf [Accessed 25 August 2016]

Evidence-Based Dentistry (2016) 17, 73-74. doi:10.1038/sj.ebd.6401182 